\title{
Tracking probability unconsciously
}

Shao-Min Hung ${ }^{1 *}$, Daw-An Wu ${ }^{1}$, Po-Jang Hsieh ${ }^{2}$, Shinsuke Shimojo ${ }^{1,3}$

${ }^{1}$ Biology and Biological Engineering, California Institute of Technology, Pasadena, CA, USA

${ }^{2}$ Department of Psychology, National Taiwan University, Taipei, Taiwan

${ }^{3}$ Computation and Neural Systems, California Institute of Technology, Pasadena, CA, USA

*Correspondence: smhung@caltech.edu 


\begin{abstract}
Extracting statistical regularities from the environment is crucial for survival. It allows us to learn cues for where and when future events will occur. Can we learn these associations even when the cues are not consciously perceived? Can these unconscious processes integrate information over long periods of time? Here we show that human visual system can track the probability of location contingency between an unconscious prime and a conscious target over a period of time on the order of minutes. In a series of psychophysical experiments, we adopted an exogenous priming paradigm and manipulated the location contingency between a masked prime and a visible target (i.e., how likely the prime location predicted the target location). The prime's invisibility was verified both subjectively and objectively. Although the participants were unaware of both the existence of the prime and the prime-target contingency, our results showed that the probability of location contingency was tracked, and manifested in the subsequent priming effect. When participants were first entrained into the fully predictive prime-target probability, they exhibited faster responses to the more predictive location. On the contrary, when no contingency existed between the prime and target initially, participants showed faster responses to the less predictive location. These results were replicated in two more experiments with increased statistical power and a fine-grained delineation of prime awareness. Together, we report that human visual system is capable of tracking unconscious contingency over a period of time, extending the known flexibility of unconscious processing.
\end{abstract}




\section{INTRODUCTION}

The influence of imperceptible external sensory stimuli upon our behavior has generally been regarded as rather automatic ${ }^{1}$, typically proceeding in a stimulus-driven and attention-free fashion. Although a few studies have shown that an unconscious stimulus can orient bottom-up attention ${ }^{2-}$ ${ }^{4}$, it is still under debate whether the processing of unconscious information requires top-down attentional resources. Recent studies have begun to show how top-down attention directs unconscious processes. For example, Kiefer and Martens $^{5}$ showed a context-dependent unconscious priming effect where a masked word elicited a stronger semantic effect if it was presented subsequent to a semantic task, as opposed to a perceptual task. This finding indicates that the top-down cognitive set can guide unconscious processes, just as it does for conscious processes. More recently, Hung et al. ${ }^{6}$ showed that unconscious semantic interference is dependent on attentional resources. Specifically, a semantic interference between an interocularly suppressed prime and a subsequent target only occurred when the task on the target was of low load but not of high load. Together, these findings paint a picture of how attentional control gates unconscious processes, contradicting attention-free accounts of subliminal processing.

Another outstanding question in the field concerns whether unconscious processing is purely stimulus-driven. Is unconscious processing merely driven by the current local stimulus, or can information from multiple unconscious stimuli spread across space and time be integrated to form an internal representation? In stark contrast to conscious processing, for which integration has been proposed as a key feature ${ }^{7,8}$, studies of information processing without consciousness are generally based on individual stimuli, lacking connectivity to the past or future. However, a few studies have recently begun to show that unconscious information can be stored temporarily and utilized subsequently. Soto et al. ${ }^{9}$ showed that a masked orientation could be maintained and used to compare with a target orientation later. Even with intervening interference from another visible orientation or a 2-second delay, the orientation of the invisible cue still affected performance. In a similar vein, Hung \& Hsieh ${ }^{10}$ showed that an interocularly suppressed sequence of words could affect a subsequent lexical decision. The results indicated syntactic integration of two unconscious words presented across several seconds, which could then prime the following visible target word based on their global syntactic congruency. These findings suggest that unconscious information 
can be held internally for a brief period, which leads to the central question of the current study: Can we integrate information over multiple unconscious stimuli over a lengthened period of time?

Here we directly tested whether humans can extract the probability of location contingency between an unconscious prime and a conscious target across dozens of presentations, over a period of several minutes. We asked: Can a change of predictive probability between an unconscious prime and a conscious target, albeit outside one's visual awareness and knowledge, alter how our visual system takes advantage of the prime? We utilized a spatial cuing paradigm (Fig. 1), with the visual prime sandwiched between a forward and a backward mask. Participants were instructed to judge the orientation of the following target, a slightly tilted Gabor patch. Unbeknown to the participants, the prime-target location contingency (i.e., how well a prime predicts the target location) was manipulated over time (Fig. 2). In the training phase, participants were exposed to an extreme cue-target contingency (either $50 \%$ or $100 \%$ ), while in the testing phase all participants were exposed to an intermediate contingency (75\%). If the training phase's predictive probability between the prime and target could be encoded despite there being no awareness of the prime, we expected to see an altered priming effect in the later trials depending on the contingency training received. 


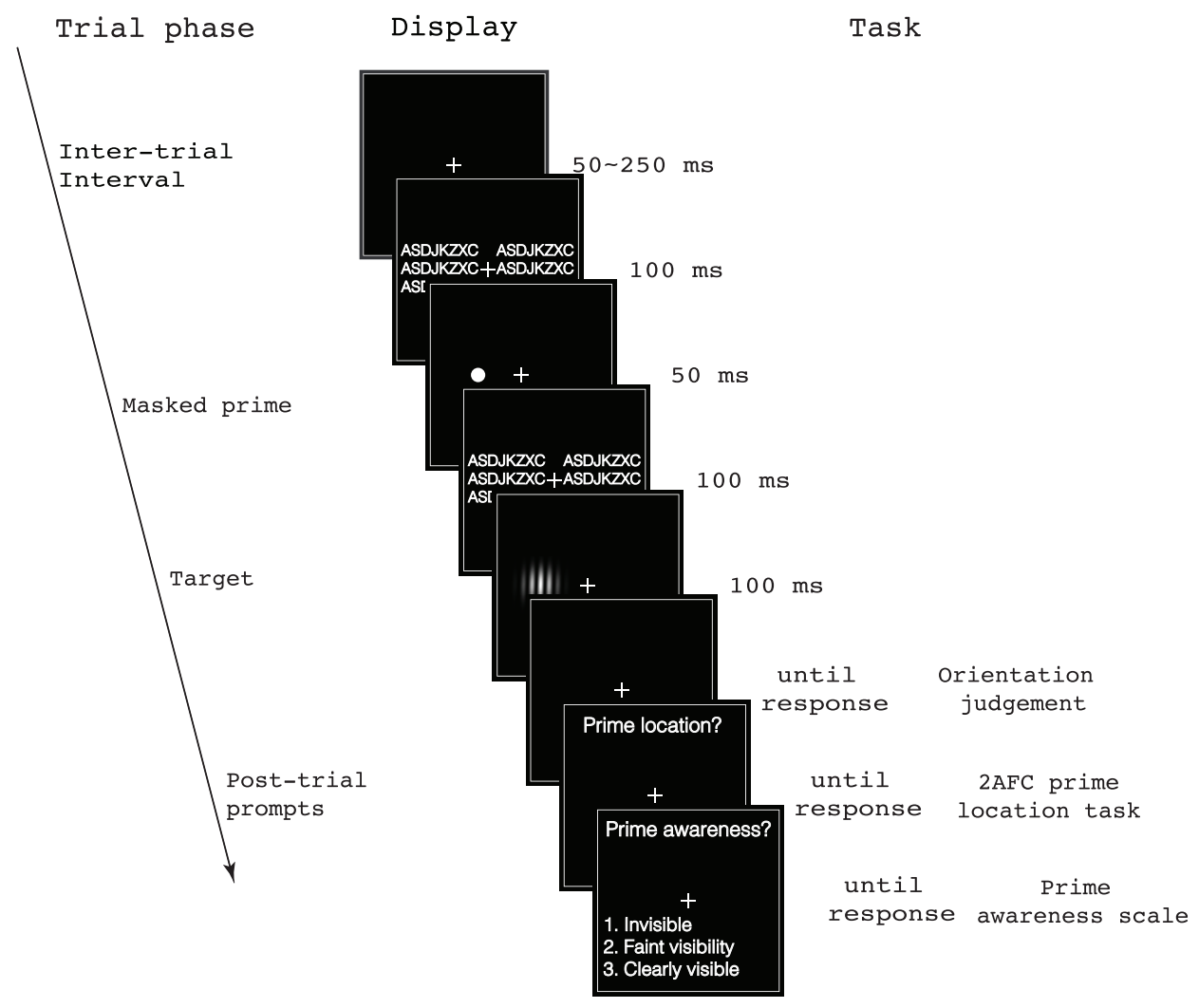

Fig. 1. Trial sequence, stimulus, and task. Each trial was preceded by a randomized ITI from 50 to $250 \mathrm{~ms}$. The trial began with a masked location prime: A dot was presented for $50 \mathrm{~ms}$, temporally sandwiched between $100 \mathrm{~ms}$ forward and backward masks consisting of meaningless letter strings. These were immediately followed by the $100 \mathrm{~ms}$ target, a tilted Gabor patch, on which an orientation judgement was made. After each trial, a 2AFC prime location task was conducted to objectively assess the prime visibility. In Experiments 3 and 4, a prime awareness scale was added to allow the participant to further categorize their subjective awareness of the prime. 


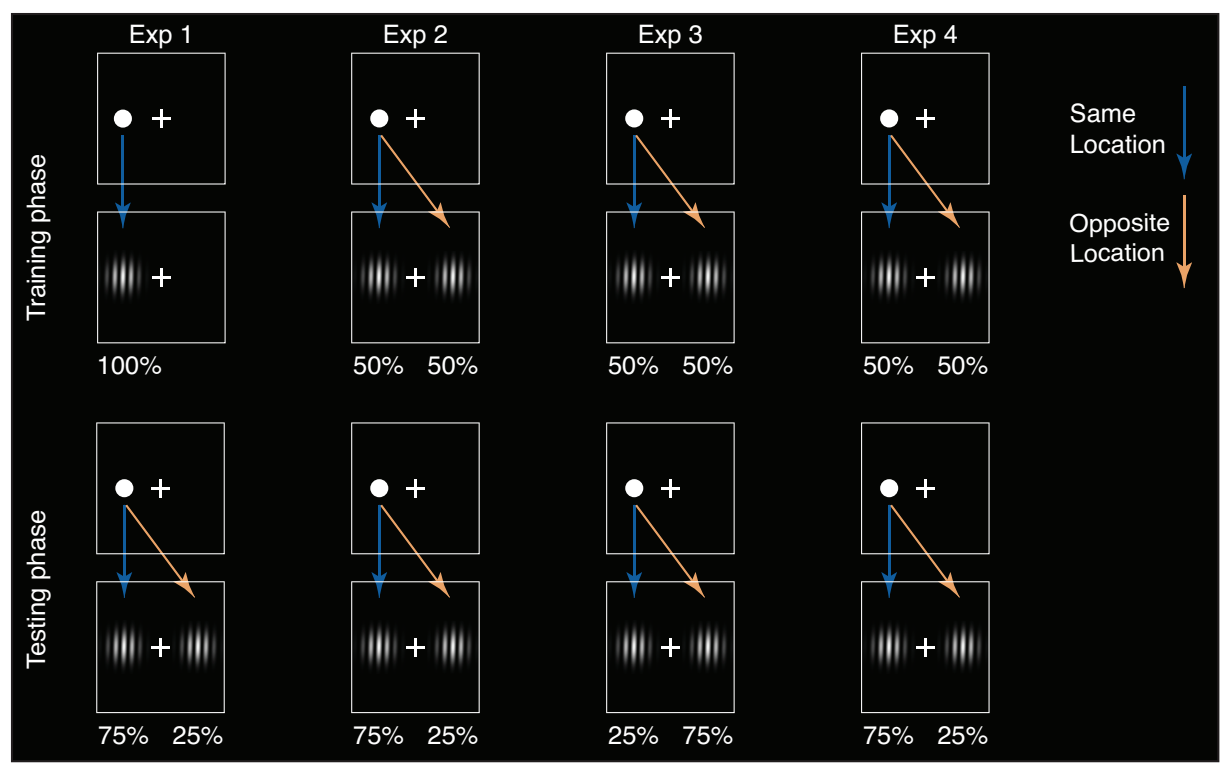

Fig. 2. Probability maps for all experiments. Each experiment had a training and a testing phase with distinct prime-target contingencies. This figure illustrates the scenario when the cue appears on the left side.

\section{RESULTS}

\section{Altering probability led to distinct unconscious spatial priming}

All numbers are reported in the format of mean (SEM). In Experiment 1, to check that the masked prime was objectively invisible, we first calculated the accuracy of the 2AFC prime location task and showed $48.40 \%(1.65 \%)$ (compared to $50 \%$, paired $t(9)=-0.97, p=0.36)$. This result indicated successful masking of prime visibility. The result in Experiment 2 did indicate above-chance localization of the prime: $52.11 \%(0.89 \%)$ with paired $t(9)=2.37, p=0.04$ when compared to $50 \%$. We thus further examined the role of prime visibility in the main prime-target congruency effect. The result showed a lack of correlation between the 2AFC location task accuracy and the congruency RT effect $(r=0.12, p=0.75)$, indicating that the priming effect could not be accounted for by prime visibility. This was further validated by more detailed prime perceptual awareness scale report in Experiments 3 \& 4 . 
In order to ensure proper fixation, we embedded catch trials $(20 \%$ of total trials) in which participants were instructed to respond to fixation cross color change as quickly and as accurately as possible once it occurred. Since all catch trials had to be responded to in order to proceed, which led to $100 \%$ accuracy, we used percentage of trials not exceeding 2 standard deviations of mean catch trial RT as a proxy of participants' sustained attention on the fixation cross. In Experiment 1, we found consistent performance in these catch trials: $97.97 \%(0.24 \%)$ of the trials were within the 2 STD threshold. Similar result was acquired in Experiment 2: $97.03 \%(0.36 \%)$.

To examine whether prime-target location congruency affected target response, we directly compared the accuracy (ACC) and reaction time (RT) when the prime and target were presented in the same location (congruent, $\mathrm{CON}$ ) vs. different locations (incongruent, INCON). Prior to these main experiments, we conducted two pilot experiments with fixed contingency between the prime and target throughout the experiment to first establish the validity of this priming paradigm. With $100 \%$ and $50 \%$ prime-target location contingency, both showed faster response times in the primecongruent location (100\%: CON: $735.3 \mathrm{~ms}(29.1 \mathrm{~ms})$ vs. INCON $746.7 \mathrm{~ms}(30.0 \mathrm{~ms}), t(8)=-2.80$, $p=.02$, Cohen's $\mathrm{d}_{\mathrm{av}}=0.39 ; 50 \%$ : CON: $729.0 \mathrm{~ms}(27.2 \mathrm{~ms})$ vs. INCON $744.6 \mathrm{~ms}(27.7 \mathrm{~ms}), t(9)$ $=-6.12, p=.0003$, Cohen's $\left.d_{\mathrm{av}}=0.57\right)$ ). However, ACC did not differ (100\%: CON: 84.64\% $(4.14 \%)$ vs. INCON 84.20\% (3.64\%), $t(8)=0.50, p=.63$, Cohen's $d_{a v}=0.11 ; 50 \%:$ CON: $90.45 \%$ $(1.81 \%)$ vs. INCON $92.01 \%(2.07 \%), t(8)=-1.73, p=.12$, Cohen's $\left.d_{a v}=0.80\right)$.

The same analyses were performed on the data of the testing phase in Experiments 1 and 2 where the testing phase ( $75 \%$ contingency) occurred subsequent to distinct training phases (Experiment 1: 100\% contingency; Experiment 2: 50\% contingency). Both experiments showed a priming effect from the unconscious prime, but in opposite directions. In Experiment 1, a paired t test showed shorter RT in the congruent trials (CON: $782.4 \mathrm{~ms}(50.1 \mathrm{~ms})$ vs. INCON $800.4 \mathrm{~ms}(52.5$ $\mathrm{ms}), t(9)=-2.97, p=.02$, Cohen's $\mathrm{d}_{\mathrm{av}}=0.35$ ). In Experiment 2, an identical analysis showed shorter RT in the incongruent trials (CON: $800.2 \mathrm{~ms}(41.6 \mathrm{~ms})$ vs. INCON $782.8 \mathrm{~ms}(38.0 \mathrm{~ms})$, $t(9)=2.88, p=.02$, Cohen's $\left.d_{a v}=0.44\right)$ (Fig. 3). Similar to our pilot experiments, ACC did not show a difference between the two conditions (Experiment 1: CON: 89.37\% (2.55\%) vs. INCON 
91.56\% (3.46\%), $t(9)=-1.02, p=.34$, Cohen's $d_{\mathrm{av}}=0.73$; Experiment 2: CON: $86.15 \%(2.01 \%)$

vs. INCON $88.12 \%(2.37 \%), t(9)=-1.10, p=.30$, Cohen's $\left.d_{a v}=0.9\right)$.
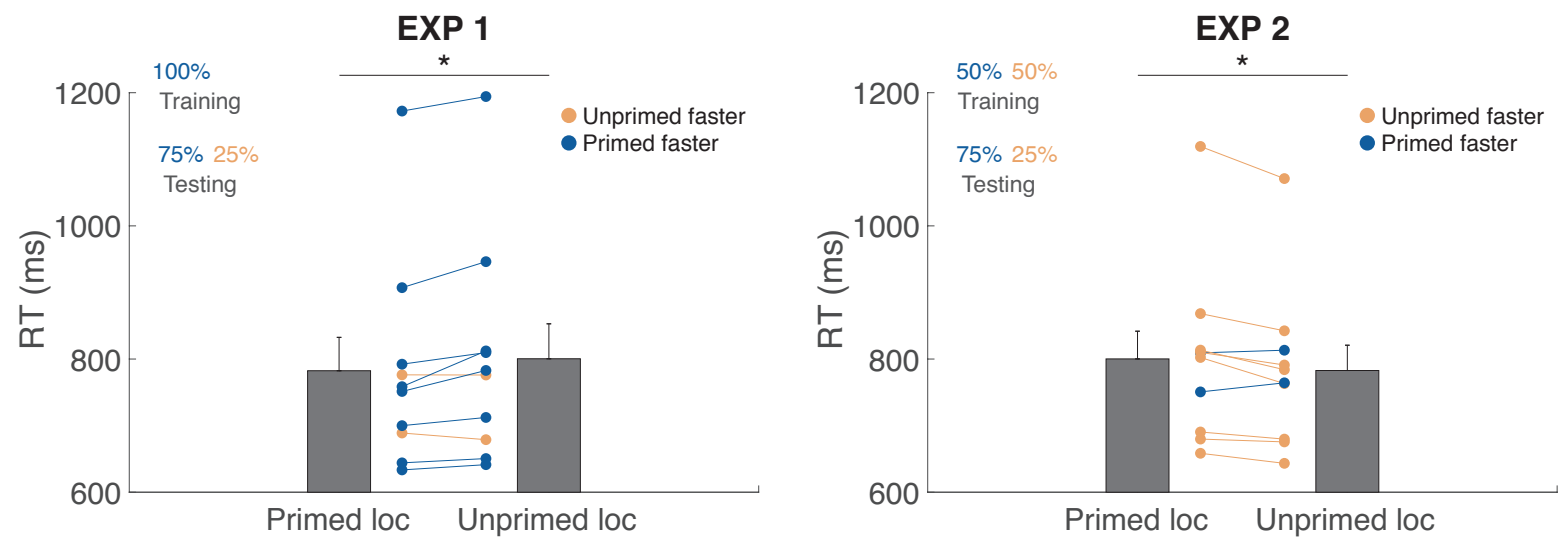

Fig. 3. Experiments 1 and 2, testing phase results. The y axis denotes reaction time (RT), and the $\mathrm{x}$ axis denotes target location with respect to the prime. The bars denote group mean with the error bars indicating standard error of the mean (SEM). Each pair of connected dots represents one participant. Navy color denotes shorter RT in the primed location while orange color denotes shorter RT in the unprimed location. Asterisk denotes $p<.05$.

These results showed that a brief training (i.e., 80 trials x 2 blocks, approximately 10 minutes) on the implicit contingency between an unconscious prime and a conscious target may induce distinct priming effects: When the prime-target was highly contingent in the training, participants later showed a primed location benefit: shorter RT to the primed location. In contrast, when the primetarget was not contingent in the training, shorter RT to the unprimed, low-contingency location was observed. The divergence of priming effects subsequent to distinct implicit prime-target contingency indicated a strong top-down contingency-tracking mechanism that overturned bottom-up saliency-based unconscious priming. A direct comparison between the RT difference of the location congruent and incongruent trials in Experiments 1 and 2 supported this reversed pattern (one sample t: $t(9)=-3.37, p=.008$, Cohen's $d=1.95$ ).

It seemed odd that the non-contingency training led to a reversal in the priming effect during the test period, rather than simply weakening or nullifying the priming as one would have expected. In experiments 3 and 4, we clarified the nature of this reversal, and explored the timecourse of its 
development. These further experiments also served to replicate and solidify the reversal result, in case it was merely a chance occurrence, or other artifact. To this end, we doubled the number of participants and increased the trials both in the training and testing periods. Now each experiment was equipped with three training blocks and three testing blocks ( 80 trials/block in Experiment 3; 120 trials/block in Experiment 4). Furthermore, we added in a perceptual awareness scale on a trial-by-trial basis for us to better delineate participants' subjective awareness of the prime (Fig. 1. More details in Methods).

\section{Low contingency attracted attention subsequent to no contingency training}

There are two potential explanations to the results in Experiment 2 where participants responded faster to the unprimed location in the testing phase. After being unconsciously trained with no

contingency between the prime and target locations, participants may have been implicitly allocating their attention to the low-contingency location (contingency-based attentional effect). On the other hand, participants may have been simply orienting their attention away from the prime or to the opposite location of the prime (prime-based attentional effect). Experiment 3 set out to tease apart these two possibilities. We reversed the contingency between the prime and the target in the testing phase so that the prime was only $25 \%$ contingent to the target location. Other changes were made to increase the power and allow participants to classify their prime awareness (see Methods for details).

Again, to establish that the masked prime was objectively invisible, we first calculated the accuracy of the 2AFC prime location task and showed 50.56\% (1.24\%) (compared to 50\%, paired $t(19)=0.45, p=0.66)$. This result indicated successful masking of prime visibility. Further, participants showed consistent performance in the catch trials: $96.82 \%(0.34 \%)$ of the trials were within the 2 STD RT threshold. Trial-by-trial perceptual awareness scale on the prime also showed that the majority $(84.45 \%)$ of the trials were categorized as "completely invisible" and only $15 \%$ trial were categorized as "faint visibility" (i.e., participants felt that they have some faint visibility of the prime but could not reliably localize it) (Fig. S1). For further analysis, we then selected the trials that were reported with (1) "completely invisible" or (2) "somewhat visible" only if the participant showed chance performance on the 2AFC location task in these trials. RT analysis sorted by prime-target location congruency showed faster RT in the congruent trials $(25 \%)$ with 
CON: $737.9 \mathrm{~ms}(20.8 \mathrm{~ms})$ vs. INCON $745.2 \mathrm{~ms}(19.5 \mathrm{~ms}), t(19)=-2.71, p=.01$, Cohen's $\mathrm{d}_{\mathrm{av}}=$ 0.36 (Fig. 4, EXP3). This result was consistent with Experiment 2 and showed that attention allocation was drawn toward the low-contingencylocation, rather than away from the cue.
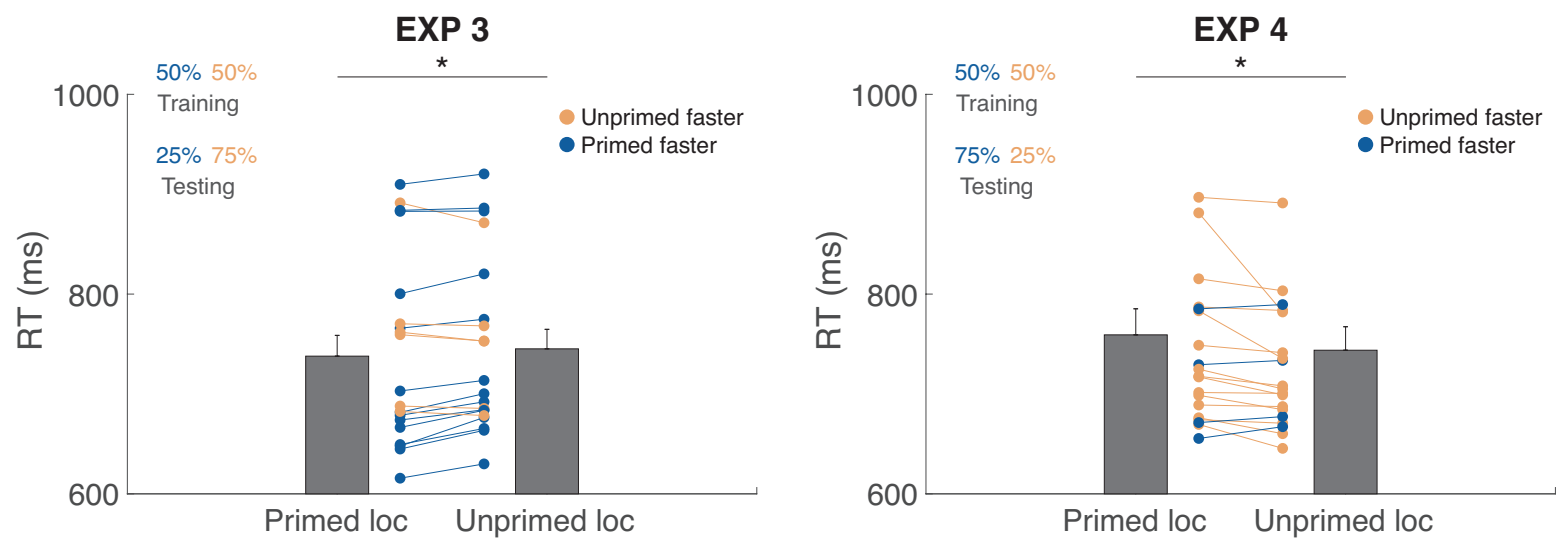

Fig. 4. Experiments 3 and 4, testing phase results. The y axis denotes reaction time (RT), and the $\mathrm{x}$ axis denotes target location with respect to the prime. The bars denote group mean with the error bars indicating standard error of the mean (SEM). Each pair of connected dots represents one participant. Navy color denotes shorter RT in the primed location while orange color denotes shorter RT in the unprimed location. Asterisk denotes $p=.01$.

\section{Further replication of the low-contingency effect and examination of timecourse}

Experiments 2 and 3 showed that after immersing in the non-contingent, unconscious prime-target relationship, subsequent low-contingency oriented participants' attention. It was unlikely that such an attentional effect was simply based on bottom-up prime saliency. For example, in Experiment 2, participants responded faster to the location opposite to the prime location, indicating that the attention distribution could be top-down and internally driven. Since this was the most critical result in our study, in Experiment 4, we set out to directly replicate it in yet another new group of participants. Experiment 4 had all test enhancements that we made in Experiment 3, including doubled number of participants, increased trial number, and inclusion of the perceptual awareness scale in every trial. Furthermore, we added blank trials in the testing period where no prime was presented as the baseline condition to allow assessment of the congruency effect direction. 
As before, we first showed that the chance accuracy of the 2AFC prime location task at 50.38\% $(1.17 \%)$ (compared to $50 \%$, paired $t(19)=0.33, p=0.75)$ ). Participants' consistent performance in the catch trials was again observed: in the RT domain, $96.77 \%(0.39 \%)$ of the trials were within the 2 STD. A majority (77.72\%) of the trials were categorized as "completely invisible", and $20.16 \%$ of the trials were categorized as "faint visibility" (Fig. S1). For further analysis, we again selected the trials that were reported with (1) "completely invisible" or (2) "faint visibility" only when the participant showed chance performance on the 2AFC location task. RT analysis sorted by prime-target location congruency revealed similar results from Experiment 2: Decreased RT in the incongruent trials (25\%) with CON: $759.2 \mathrm{~ms}(26.1 \mathrm{~ms})$ vs. INCON $743.8 \mathrm{~ms}(23.5 \mathrm{~ms}), t(19)$ $=2.70, p=.01$, Cohen's $\mathrm{d}_{\mathrm{av}}=0.62$ (Fig. 4, EXP4). Although the mean RT of the blank trials fell between that of CON and INCON trials: $751.0 \mathrm{~ms}(21.3 \mathrm{~ms})$, the difference did not reach significance $\left(\mathrm{CON}\right.$ vs. blank $t(19)=1.30, p=.21$, Cohen's $\mathrm{d}_{\mathrm{av}}=0.35$; INCON vs. blank, $t(19)=$ $1.50, p=.15$, Cohen's $\mathrm{d}_{\mathrm{av}}=0.32$ ). Experiment 4 thus successfully replicated the results of Experiment 2, with more participants, a larger number of trials, and resulting in an increased statistical significance and effect size.

To assess the temporal dynamics of the priming effect, we further conducted a two-way repeated measures analysis of variance on the RT with prime-target congruency and block number as the factors. A marginal main effect of block was found, $F(1,19)=2.18, p=.06, \eta_{\mathrm{p}}^{2}=0.1$. Furthermore, there was an interaction between congruency and block, $F(1,19)=2.14, \mathrm{p}=.07, \eta_{\mathrm{p}}{ }^{2}$ $=0.1$. Post-hoc comparisons showed marginal effects on priming only in the first block of the training phase $(t(19)=-2.07, p=.05)$ and in the final two blocks of the testing phase $(t(19)=2.80$, $p=.01 \& t(19)=2.35, p=.03)$. Critically, the initial priming effect, attributable to the default exogenous cuing, was reversed across the training and testing phases (Fig. 5), reflecting a gradual process that acted to nullify the exogenous cuing effect during the training phase, and then acted further during the testing phase, ultimately leading to a fully reversed cuing effect in the last two blocks. 


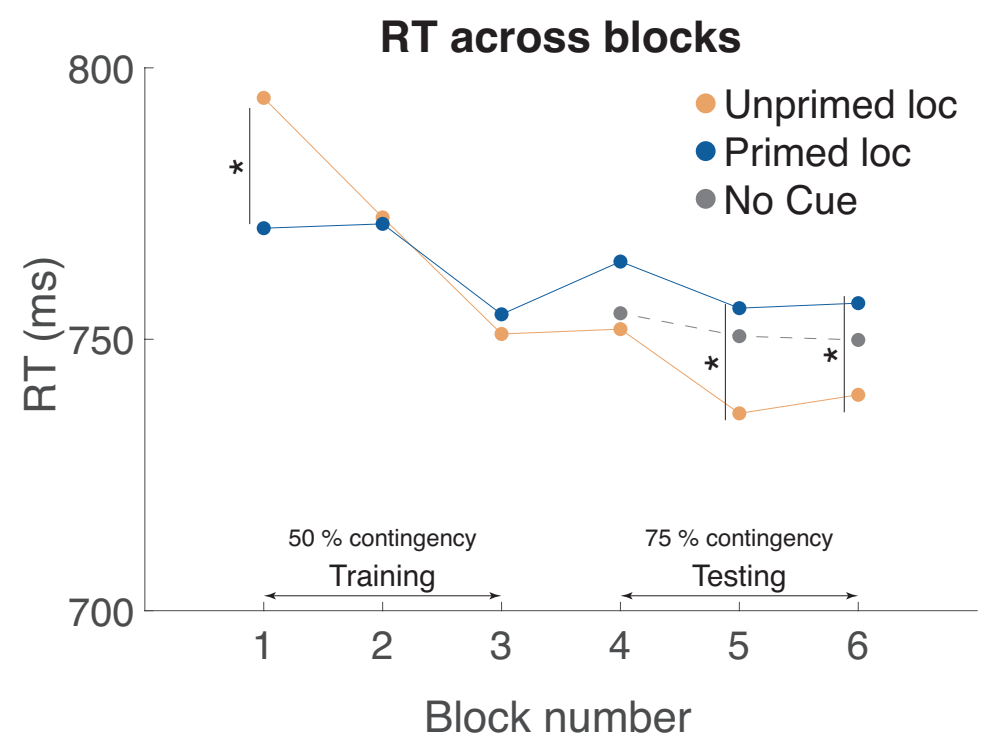

Fig. 5. Temporal dynamics of the priming effect in Experiment 4. The typical location-based congruency priming was found in the first training block and decreased over time. Distinctively, the decreased RT in the unprimed, low-contingency location was found in the later blocks. Asterisk denotes $p<.05$. 


\section{GENERAL DISCUSSION}

We used a series of psychophysical experiments to show unconscious probability tracking in the human visual system. An unconscious location priming effect was guided by the prime-target predictive probability that participants were exposed to over a period of time in the training lasting approximately 10 minutes. A direct comparison of the testing phases in Experiments 1 and 2 showed that the pattern of spatial attention triggered by the prime was dependent on the primetarget contingency contained in the training period. These findings reveal the capacity of our visual system to integrate unconscious information over multiple incidences and a period of time on the order of minutes. Critically, an internal contingency map needs to be formed and updated throughout the experiment to track the frequency of co-occurrences between the prime and the target, supporting a dynamic and flexible unconscious system.

In addition, the particular pattern of priming that emerged was interesting. When the prime effectively predicted target location during the training, participants in subsequent trials responded faster to the primed location. In contrast, when the prime was not predictive of the target location, a faster reaction time was found in the low-contingency location. Two additional experiments further replicated the results of Experiment 2 with increased statistical power, again revealing faster reaction times in the low-contingency location after non-contingency training. The temporal profile of this shift toward the low-contingency location suggested a slow process spanning the whole experiment, where the attention allocation triggered by the prime slowly shifted to the location with low cue-target contingency.

The results in the current study can be considered in the context of the typical location-priming paradigm $^{1}$, in which a preceding prime orients the observer's attention in a bottom-up, exogenous manner. Past studies have consistently documented such a priming effect with conscious cues ${ }^{11}$ $\left(\right.$ see review ${ }^{12}$ ) or unconscious cues ${ }^{13}$ (see review ${ }^{14}$ ). However, simple stimulus-based saliency or exogenous attention capture can explain neither the essential nor the curious aspects of our results.

First of all, our results showed that unconscious priming could be independent of or even against mere stimulus saliency (e.g., the more salient spatial location), in a manner dependent on the cue- 
target contingencies present in the training period. The behavioral divergence between Experiments 1 and 2 thus requires an endogenous factor which is formed as a reaction to the integrated statistical properties of temporally scattered subliminal cues.

Secondly, and curiously, training with no cue-target contingency led to a situation where, with an increased physical contrast in the primed location that was supposed to attract attention, participants' attention was not only nulled, but in fact deployed to the unprimed location. It is worth noting that the no-contingency training does not simply cause attention to be deployed away from subsequent cues, but away from subsequent high-contingency locations. Thus, in Experiment 3 , reactions times were faster to the cued location, where targets were less frequent. The temporal data from Experiment 4 (Fig. 5) provides more detail as to how this reversal pattern forms. There is a gradual learning process whereby the initial separation in reaction times (attributable to an exogenous priming effect) becomes nulled during the training with non-contingent cues. This nulled state continues for some time into the test period, even though a contingency has been introduced. With sufficient exposure to the new contingency, the priming effect shifts to the lowcontingency location. The reversal of the total priming effect indicates that a weakening of exogenous priming would not suffice as an explanation. Instead, there appears to be a build-up of a countervailing endogenous priming process. This is consistent with the descriptive aspects of the timecourse data, in which the reaction time to the primed (exogenous) location remains fairly flat, while the reaction time to the unprimed location falls, not only in the training phase, but also into the testing phase. A full accounting of this additional learning that leads to reversal is beyond the scope of this study. But this clearly reinforces the idea that the statistics of the subliminal cues are continually being integrated, and that the endogenous processing continues to be updated as the statistical landscape changes. Further research is needed to clarify what those processes are. In the meantime, it may be worthwhile to entertain some speculative accounts and connections to similarly-shaped findings, to bring to light some interesting possibilities.

One possible form of learning that may have been occurring during the testing phase to this attraction to low-contingency locations, is that after exposure to unpredictable primes in training, the rare events (i.e., the low-contingency targets) in testing became salient and captured attention. To grasp the idea of scarcity, a continuous tracking of prime-target contingency is required. 
Critically, the priming effect in the testing phase required time to build up and only occurred in the later blocks, indicating an accumulation of prime-target statistics.

The unconscious contingency learning observed in the current study likely resulted from implicit learning rather than explicit strategic learning. Given the verified objective and subjective prime invisibility, participants were oblivious both to the existence of the prime and the prime-target relationship, with the latter confirmed by all participants in the debriefing. At least one prior study has examined whether a seemingly automatic response was triggered by reflexive response, implicit learning, or planned strategy utilizing a go/no-go paradigm ${ }^{16}$. In a typical go/no-go paradigm, participants exhibit the post-stop-signal slowing, reflecting a typical conflict between executing a go response and suppressing a response upon recently receiving the no-go signal. Bissett and Logan ${ }^{16}$ showed that the post-stop-signal slowing was not due to reflexive response but instead could be driven by implicit learning or a shift in strategy. One particular result revealed strong implicit learning: when participants were oblivious to the contingency between the current no-go trial and the subsequent one, they nevertheless exhibited post-stop-signal slowing when the no-go trial had a higher contingency of preceding another no-go trial (i.e., two consecutive no-go trials). This phenomenon based on implicitly accumulating the contingency of no-go trials was observed in the later stage of the experiment, suggesting gradual learning. Our study provided an even more "unconscious" scenario where the contingency of the prime and the subsequent target was not only unbeknown to the participants, but the prime was also subliminal, which revealed a novel unconscious contingency learning over time.

It is worth pointing out that our results do not endorse a full-on flexible unconscious system that can adaptively select, maintain, and suppress information based on goal-directed behavior. It is apparent that the participants in the current study were still passively receiving and updating the probability between an unconscious prime and a conscious target. No active and deliberate topdown choices were made to deploy their attention accordingly. In fact, it is the lack of intention and awareness of the prime that made our findings particularly intriguing. The fact that our data show contingency tracking over many trials without the observers (1) seeing the prime and (2) knowing the prime-target relationship informs how well our visual system extracts statistical regularities from the environment. 
These results can be surprising at first glance but not so much when considered in the evolutionary context. Extracting statistical regularities from the sensory inputs in the wild is deemed an important survival skill both for predators and prey. It is thus not surprising to see that non-human primates, such as cotton-top tamarins ${ }^{17,18}$ and marmosets/macaques ${ }^{19}$, exhibit the ability to learn abstract language-like syntactic patterns. More rudimentary tracking of statistical regularities, such as detection of frequencies of co-occurrences or transitional probabilities can be found in songbirds (Zebra finches ${ }^{20}$ ) and newborn domestic chicks ${ }^{21}$. Human neonates are able to detect transitional probabilities at ages as young as 4.5 months old ${ }^{22}$. The cross-species evidence together suggests that a core mechanism to track probabilities among sensory items is developed early in ontogeny and evolution. In fact, a century-long hypothesis ${ }^{23}$ that single-cell organism (i.e., ciliate Stentor roeseli) exhibits a rudimentary form of learning by showing avoidant behavior to aversive stimuli has been recently replicated ${ }^{24}$. Such results suggest that monitoring stimulations in the external environment while forming some internal storage has an astonishingly long history (a recent review $\left.^{25}\right)$.

Coming back to humans, the limited bandwidth of conscious vision has been repeatedly shown ${ }^{26}$, with multiple bottlenecks such as attention and photoreceptor density. If an ability fundamental to our life, such as integrating statistical regularities in our environment, is limited to conscious vision, it is hard to conceive how the massive amount of information can be dealt with moment by moment. Here we provide a possible solution: unconscious integration of probability over multiple incidences. Our findings thus provide novel evidence to support an active machinery where cooccurrences of individual visual items were unconsciously tracked over a period of time, revealing the adaptive values of our unconscious visual system. 


\section{METHODS}

\section{General experimental apparatus}

In all experiments, the visual stimuli were generated with MATLAB (The MathWorks, Inc., Natick, MA) and PsychToolbox ${ }^{27,28}$. Participants viewed the monitor while resting on a chin rest from a distance of $57 \mathrm{~cm}$. The stimuli were presented against a black background on a 21.5-in. iMAC LCD monitor with a resolution of $1,920 \times 1,080$ pixels and a refresh rate of $60 \mathrm{~Hz}$.

\section{Participants}

All participants (age range: 19-37) reported normal or corrected-to-normal vision. They reported no history of psychological disorders and were not on medication at the time of experiment. They gave written informed consent prior to the experiment and were reimbursed $\$ 5 / \$ 10$ for participating in a 30-min (Experiments 1-3) or a 45-min (Experiment 4) session. This study was approved by the institutional review board of National Taiwan University and the California Institute of Technology. Participants that (1) had accuracy rates 3 standard deviations away from the group mean on the main task or/and (2) figured out the experimental design (3) failed to achieve desired pre-experiment prime contrast calibration (i.e., chance-rate localization on prime) were removed before entering analysis. (see Stimuli and Procedure of each experiment; Experiment 1: $n=4$; Experiment 2: $n=2$; Experiment 3: $n=4$; Experiment 4: $n=7$ ). Experiments 1 and 2 aimed at 10 participants based on our pilot experiments $(n=9$ for both experiments). Experiments 3 and 4 further increased sample size and recruited 20 participants each. This decision was based on the power calculation from Experiment 1 data and was rounded to the nearest 10. All participants were naïve to the purpose of the experiments.

\section{Reaction time data pre-analysis processing}

All trials that had reaction time shorter than $200 \mathrm{~ms}$ were pre-excluded. Furthermore, the reaction time data underwent per-participant per-condition outlier removal to remove data points 2 standard deviations away from the average.

\section{Pre-experiment prime thresholding procedure}


In each experiment, participants first completed a pre-experiment thresholding procedure on prime contrast in which they simply judged the location of the masked prime. This was aimed to acquire individual prime contrast level that guaranteed objective unconsciousness of the prime. The procedure was identical to that of the main experiment. In each trial, after a varied ITI ranging from 50 to $250 \mathrm{~ms}$, a prime disc $\left(0.5^{\circ}\right)$ was presented to the left or right of the fixation point for approximately $50 \mathrm{~ms}$ and was sandwiched temporally between two 100 -ms noise patterns made of random letters. Participants had to judge the location immediately after prime presentation. The prime started with $20 \%$ of the full alpha value, which was entirely visible, and followed a 1-up-1down procedure thresholding staircase: if the prime location was correctly reported, the contrast decreased by $3 \%$ of the full alpha value in the next trial; otherwise if the location was incorrectly reported, the contrast increased by 3\%. The thresholding procedure contained 40 trials, and the final contrast level was taken to the main experiment.

\section{Experiment 1: experimental design and procedure}

Four blocks with each containing 80 trials were completed in the main experiment. Unbeknown to the participants, the first two blocks were the training blocks, and the last two blocks the testing blocks. Each trial began with a varied SOA ranging from 50 to $250 \mathrm{~ms}$. Identically to the thresholding procedure, the prime disc $(50 \mathrm{~ms})$ was presented to the left or right of the fixation point and temporally sandwiched by two noise patterns (100 ms each). Subsequently, a Gabor patch served as a target and lasted $100 \mathrm{~ms}$. The target was tilted clockwise or counterclockwise for 2 degrees. Participants were asked to first judge the tilt orientation of the Gabor patch as accurately and as quickly as possible and later reported the location of the prime in a two-alternative-forcechoice (2AFC) task. Prime location, target location, and target orientation were counterbalanced. The prime-target location congruency was set at 100\% (i.e., always on the same location) in the training blocks and at 75\% (i.e., on the same location 3 out of 4 times) in the testing blocks. To encourage proper fixation, we embedded catch trials in which the participants had to respond promptly when the fixation color changed. These catch trials comprised $20 \%$ of all the trials. A trial sequence illustration is provided in Fig. 1.

\section{Experiment 2: experimental design and procedure}


The design and procedure of Experiment 2 was identical to that of Experiment 1, except that the prime-target location congruency in the training blocks was set at 50\% (i.e., the prime had no predictive power of the target). Testing blocks were unchanged from Experiment 1, with 75\% contingency.

\section{Experiment 3: experimental design and procedure}

The design and procedure of Experiment 3 was identical to that of Experiment 2 except for the following changes in the main experiment. (1) The prime-target location contingency was set at $25 \%$ in the testing blocks. (2) At the end of the trial, a perceptual awareness scale ${ }^{29}$ was added for the participants to report their awareness of the prime with one of the following categories: Not seen at all (Invisible)/Seen but not sure where it was (Faint visibility)/ Seen clearly (Visible). (3) The block number of both the training and the testing phases was increased to three, resulting in a total of 480 trials.

\section{Experiment 4: experimental design and procedure}

The design and procedure of Experiment 4 was identical to that of Experiment 2 except for the following changes in the main experiment. (1) A perceptual awareness scale was added at the end of each trial to gauge prime visibility. (2) The trial number in each block was increased to 120 , resulting in a total of 720 trials. (3) Blank trials where no prime was present (while the masks were still presented) were added in the testing phase. The ratio of congruent:blank:incongruent trials was $3: 2: 1$. 


\section{REFERENCES}

1. Posner, M. I. \& Snyder, C. R. R. Attention and Cognitive Control. in Information Processing and Cognition: The Loyola Symposium (ed. Solso, R. L.) (Lawrence Erlbaum, 1975).

2. Zhaoping, L. Attention capture by eye of origin singletons even without awareness-A hallmark of a bottom-up saliency map in the primary visual cortex. Journal of Vision $\mathbf{8}, 1$ (2008).

3. Hsieh, P.-J., Colas, J. T. \& Kanwisher, N. Pop-Out Without Awareness: Unseen Feature Singletons Capture Attention Only When Top-Down Attention Is Available. Psychol Sci 22, $1220-1226(2011)$.

4. Hung, S.-M., Nieh, C.-H. \& Hsieh, P.-J. Unconscious processing of facial attractiveness: invisible attractive faces orient visual attention. Sci Rep 6, 37117 (2016).

5. Kiefer, M. \& Martens, U. Attentional sensitization of unconscious cognition: Task sets modulate subsequent masked semantic priming. Journal of Experimental Psychology: General 139, 464-489 (2010).

6. Hung, S.-M., Wu, D.-A. \& Shimojo, S. Task-induced attention load guides and gates unconscious semantic interference. Nature Communications 11, 2088 (2020).

7. Dehaene, S. \& Changeux, J.-P. Experimental and Theoretical Approaches to Conscious Processing. Neuron 70, 200-227 (2011).

8. Oizumi, M., Albantakis, L. \& Tononi, G. From the Phenomenology to the Mechanisms of Consciousness: Integrated Information Theory 3.0. PLoS Comput Biol 10, e1003588 (2014).

9. Soto, D., Mäntylä, T. \& Silvanto, J. Working memory without consciousness. Current Biology 21, R912-R913 (2011). 
10. Hung, S.-M. \& Hsieh, P.-J. Subliminal temporal integration of linguistic information under discontinuous flash suppression. Journal of Vision 21, 27 (2021).

11. Posner, M. I., Snyder, C. R. \& Davidson, B. J. Attention and the detection of signals. Journal of Experimental Psychology: General 109, 160-174 (1980).

12. Egeth, H. E. \& Yantis, S. VISUAL ATTENTION: Control, Representation, and Time Course. Annu. Rev. Psychol. 48, 269-297 (1997).

13. McCormick, P. A. Orienting attention without awareness. J. exp. psychol. Hum. percept. perform 23, 168-180 (1997).

14. Mulckhuyse, M. \& Theeuwes, J. Unconscious attentional orienting to exogenous cues: A review of the literature. Acta Psychologica 134, 299-309 (2010).

15. Itti, L. \& Koch, C. Computational modelling of visual attention. Nat Rev Neurosci 2, 194$203(2001)$

16. Bissett, P. G. \& Logan, G. D. Post-stop-signal slowing: Strategies dominate reflexes and implicit learning. Journal of Experimental Psychology: Human Perception and Performance 38, 746-757 (2012).

17. Fitch, W. T. \& Hauser, M. D. Computational Constraints on Syntactic Processing in a Nonhuman Primate. 303, 4 (2004).

18. Saffran, J. et al. Grammatical pattern learning by human infants and cotton-top tamarin monkeys. Cognition 107, 479-500 (2008).

19. Wilson, B. et al. Auditory Artificial Grammar Learning in Macaque and Marmoset Monkeys. Journal of Neuroscience 33, 18825-18835 (2013).

20. Chen, J. \& ten Cate, C. Zebra finches can use positional and transitional cues to distinguish vocal element strings. Behavioural Processes 117, 29-34 (2015). 
21. Santolin, C., Rosa-Salva, O., Vallortigara, G. \& Regolin, L. Unsupervised statistical learning in newly hatched chicks. Current Biology 26, R1218-R1220 (2016).

22. Marcovitch, S. \& Lewkowicz, D. J. Sequence learning in infancy: the independent contributions of conditional probability and pair frequency information: Frequency and conditional probability learning in infancy. Developmental Science 12, 1020-1025 (2009).

23. Jennings, H. S. Behavior of the Lower Organisms. (Columbia University Press, The Macmillan Company, agents, 1906).

24. Dexter, J. P., Prabakaran, S. \& Gunawardena, J. A Complex Hierarchy of Avoidance Behaviors in a Single-Cell Eukaryote. Current Biology 29, 4323-4329.e2 (2019).

25. Gershman, S. J., Balbi, P. E., Gallistel, C. R. \& Gunawardena, J. Reconsidering the evidence for learning in single cells. eLife 10, e61907 (2021).

26. Cohen, M. A., Dennett, D. C. \& Kanwisher, N. What is the Bandwidth of Perceptual Experience? Trends in Cognitive Sciences 20, 324-335 (2016).

27. Brainard, D. H. The Psychophysics Toolbox. Spatial Vis 10, 433-436 (1997).

28. Pelli, G. Denis. The VideoToolbox software for visual psychophysics: transforming numbers into movies. Spatial Vision 10, 437-442 (1997).

29. Ramsøy, T. Z. \& Overgaard, M. Introspection and subliminal perception. Phenomenology and the Cognitive Sciences 3, 1-23 (2004). 


\section{Author contributions}

Conceptualization, S.-M.H. and D.-A.W.; Methodology, S.-M.H.; Software, S.-M.H.; Investigation, S.-M.H.; Writing - Original Draft, S.-M.H.; Writing - Review \& Editing, S.-M.H., D.-A.W., P.-J. H., and S. S.; Supervision, P.-J. H. and S. S.

\section{Acknowledgements}

We are grateful for the research funding provided by the James Boswell Postdoctoral Fellowship and the Caltech BBE Divisional Postdoctoral Fellowship to S.-M.H. and by the Yushan Young Scholar Program (NTU-108V0202) and Taiwan Ministry of Science and Technology (109- 2410H-002-004-MY3) to P.-J. H. and by the National Institute of Health to S. S. (R01AG063857).

\section{Declaration of competing interest}

The authors declare that they have no known competing financial interests or personal relationships that could have appeared to influence the work reported in this paper. 